\title{
Points aveugles et images différées : les optiques narratives dans Triptyque de Claude Simon
}

\section{Andrea Goulet}

Traducteur : Annelise Koskas et David Zemmour

\section{(e) OpenEdition \\ Journals}

Édition électronique

URL : http://journals.openedition.org/ccs/1014

DOI : $10.4000 /$ ccs. 1014

ISSN : 2558-782X

Éditeur :

Presses universitaires de Rennes, Association des lecteurs de Claude Simon

Édition imprimée

Date de publication : 1 décembre 2017

Pagination : 79-102

ISBN : 978-2-7535-5482-5

ISSN : $1774-9425$

Référence électronique

Andrea Goulet, «Points aveugles et images différées : les optiques narratives dans Triptyque de Claude Simon », Cahiers Claude Simon [En ligne], 12 | 2017, mis en ligne le 07 décembre 2018, consulté le 12 février 2020. URL : http://journals.openedition.org/ccs/1014 ; DOI : 10.4000/ccs.1014 


\title{
POINTS AVEUGLES ET IMAGES DIFFÉRÉES : LES OPTIQUES NARRATIVES DANS TRIPTYQUE DE CLAUDE SIMON ${ }^{1}$
}

\author{
Andrea GOULET \\ University of Pennsylvania
}

\section{ORION AVEUGLE}

«Tu sembles privilégier de plus en plus l'image, la description. [...] Il y aurait donc une sorte de coïncidence entre percevoir et écrire ${ }^{2}$ ? " À cette question de Lucien Dällenbach, Claude Simon répond par l'affirmative, laissant entendre que le processus d'écriture a pour lui autant à voir avec la perception visuelle qu'avec son absence, le «tâtonnement [...] à l'aveugle » d'un Orion aveugle. De fait, Simon termine Orion Aveugle par une évocation saisissante de la perception visuelle: le dessin d'un oil associé à la description d'images glissant sur la membrane rétinienne. On pourrait considérer que cette vision tend à constituer l'immédiateté de la perception en horizon vers lequel tend le texte simonien ${ }^{3}$. Mais cette représentation très particulière de l'organe de la vue justifie également qu'on y regarde de plus près, en s'appuyant sur les apports de la science optique, car aucune définition simple de la perception

1. Article originellement publié en anglais sous le titre « Blind spots and afterimages: the narrative optics of Claude Simon's Triptyque» dans Romanic Review, n ${ }^{\circ}$ 91, mai 2000, p. 289-311. Trad. Annelise Koskas et David Zemmour, revue par l'auteure. Dans la suite, l'astérisque signale les termes et expressions employés en français dans le texte.

2. L. Dällenbach, Claude Simon, Le Seuil, 1988, p. 172.

3. Pour les commentaires de Simon sur le fossé irréductible qui sépare la logique spatiale de la vision et la temporalité linéaire du récit, voir, par exemple, "La sensation, c’est primordial ", entretien de Philippe Sollers avec Claude Simon, Le Monde des livres, vendredi 19 septembre 1997. 
visuelle - comme immédiate, subjective ou non narrative - ne permet de caractériser convenablement son rôle dans les romans de Simon.

À l'avant-dernière page d'Orion aveugle, le lecteur tombe sur une planche anatomique pleine couleur représentant une tête humaine vue de profil [voir dossier iconographique, p. I]. La peau en a été retirée pour laisser voir les entrelacs épais de faisceaux musculaires reliant le lobe crânien à la mâchoire, le menton à l'œsophage, l'oreille à la poitrine. La structure osseuse des orbites et des lobes émerge, pâle, parmi les faisceaux striés de muscle rouge, et disparaît à nouveau dans les plis de la chair blanche au niveau des épaules et de la poitrine de l'homme. La tête est légèrement inclinée vers la gauche, et un grand lambeau de muscle excisé pend au-devant de l'arête du nez, partiellement détaché de l'orbite situé en-dessous. Et depuis le creux sombre de la cavité oculaire, la forme sphérique d'un œil regarde fixement, relié par des nerfs blanchâtres à la masse osseuse qui l'entoure. Un point de lumière sur la pupille de l'œil le fait paraître actif et vivant, comme si l'homme regardait depuis le tréfonds de sa chair écorchée. D'après les notes de Simon, cette image anatomique provient d'une gravure trouvée dans un manuel scientifique du dix-huitième siècle; son titre, "Tête d'homme - document tiré de "Myologie complète en couleurs et grandeur naturelle" de Jacques Gautier d'Agoty ", fait entrer l'image repoussante dans le discours en principe neutre de la science. Conformément à la vocation abstraite et objective de la présentation scientifique, la gravure désigne avec précision différentes parties du corps avec lettres et chiffres - des $a$ et des $b$ minuscules autour de l'œil, un $g$ minuscule sur l'oreille et un $G$ majuscule sur les lèvres, de petits chiffres sur les muscles. Mais l'abstraction classificatoire du diagramme est contestée par sa corporéité vivante. Sur la reproduction en couleurs, les muscles apparaissent rouge sang, en contraste avec les tons rose-blanc de la peau et des os; l'épaule ressemble à un moignon amputé et l'ensemble que forment les yeux et la bouche confèrent à la tête une expression troublante qui fait penser à un être vivant plutôt qu'à un modèle anatomique neutre.

L'étrange juxtaposition sur la gravure du tissu vivant avec la notation de manuel scolaire trouve son pendant dans la description textuelle qui termine Orion aveugle:

Une coupe longitudinale de la tête de profil permet de voir les principaux organes, la masse ivoire du cerveau injecté de sang dont les circonvolutions compliquées battent à chaque afflux, la langue violette, les dents, les os poreux et la boule exorbitée de l'œil, livide, enserrée dans ses racines rouges, avec son iris, son cristallin, son corps vitreux, et 
la mince membrane de la rétine sur laquelle les images du monde viennent se plaquer, glisser, l'une prenant la place de l'autre. (OA, p. 146)

Tout en évoquant une masse saillante et sanglante de tissus corporels, Simon utilise également des termes anatomiques précis pour décrire les composantes de l'œil: iris, cristallin, corps vitreux, rétine - des termes relevant de la physiologie oculaire qui divise l'organe selon ses diverses fonctions. Il y a, dans cette description, une conjonction frappante entre le flux vital (de sang, d'images saisies sur la surface rétinienne) et l'inévitable absence de vie d'une tête tranchée. De la coupe transversale au sujet vivant, le lien est établi par l'œil, dont la rétine continue d'enregistrer le monde extérieur, et dont le globe demeure à moitié enchâssé dans son orbite, relié au corps par ses " racines rouges » musculaires.

De la sorte, l'organe visuel agit comme un lieu de changement de registre, de l'objectivité à la subjectivité, de l'abstraction à la corporéité. Non seulement l'image finale d'Orion aveugle marque le primat de la perception visuelle dans le projet romanesque simonien, mais elle interroge aussi la nature même de la perception visuelle. En alliant précision anatomique et palpitation organique, l'œil simonien est saisi entre l'abstraction du discours et la corporéité de l'expérience individuelle - une opposition qui synthétise la distinction entre une optique cartésienne objective et classique d'une part, et l'étude subjective de la vision d'un point de vue phénoménologique d'autre part. Mais Simon va au-delà de ces deux pôles, investissant une zone intermédiaire ou "floue* " entre Esprit ${ }^{*}$ (catégorisation abstraite) et $\mathrm{Eiil}^{*}$ (perception directe ${ }^{4}$ ), une zone que l'on peut reconnaître dans ces lignes d'Histoire:

l'esprit (ou plutôt: encore l'œil, mais plus seulement l'œil, et pas encore l'esprit: cette partie de notre cerveau où passe l'espèce de couture, le hâtif et grossier faufilage qui relie l'innommable au nommé) (Hist., p. 328)

Dans l'intervalle qui sépare langage et vision, une « espèce de couture » trace imparfaitement son chemin. La fin d'Orion aveugle suggère une image possible de cette couture: la rétine elle-même, "la membrane mince de [1]a rétine sur laquelle les images du monde viennent se plaquer, glisser, l'une prenant la place de l'autre ". Concluant un roman entièrement composé d'une série de scènes au présent enchevêtrées les unes dans les autres comme des "images du Monde ", ces derniers mots retissent le lien qui va de la référence anatomique (comment fonctionne une rétine) à l'autoréférence

4. J'utilise ici les termes de Maurice Merleau-Ponty. Voir L'CEil et l'esprit, Gallimard, 1964. 
textuelle (comment fonctionne Orion aveugle). En tant que point de contact entre le sujet et les images du monde, l'écran membraneux de la rétine - ni totalement transparent ni totalement opaque - opère comme "couture » entre la perception et la représentation.

\section{L'EIL DU LAPIN (I)}

L'image rétinienne à laquelle Simon se réfere à la fin d'Orion aveugle a une histoire importante dans le domaine de l'optique. En 1604, Johannes Kepler décrit la manière dont les rayons lumineux réfractés produisent sur l'écran rétinien des images du réel, une découverte qui pour Gérard Simon constitue « bel et bien une coupure épistémologique dans l'histoire de l'optique $^{5}$ ». La théorie keplerienne des images rétiniennes, dont les fondements sont toujours valables aujourd'hui, était novatrice dans la distinction qu'elle opérait entre sujet et objet, entre l'œil comme instrument de perception et le monde extérieur soumis à des lois géométriques et physiques; en cela, il a ouvert la voie à une rationalité cartésienne qui sépare strictement l'âme et le corps, le physique et le psychique, le sujet et le monde ${ }^{6}$. Plus spécifiquement, la théorie de l'image rétinienne a valu une certaine fortune à la comparaison entre l'œil humain et la camera obscura, ce dispositif optique par lequel des images sont projetées à travers un petit trou sur un mur enclos ou un écran. Depuis Kepler, l'analogie de la camera obscura a traditionnellement été associée à une théorie de la vision qui définit la perception comme une fonction mécanisée et neutre. Sous l'influence de Descartes, ce modèle de la camera obscura en est venu à impliquer un observateur abstrait et désincarné, un observateur capable de dépasser l'opacité et la subjectivité de l'œil humain afin d'accéder à une relation au monde qui soit transparente et purement objective ${ }^{7}$.

5. G. Simon, Sciences et savoirs aux XVI et XVII siècles, Villeneuve d'Ascq, PU du Septentrion, 1996, p. 83.

6. Ibid., p. 80-84.

7. Pour aller plus loin, voir J. Crary, Techniques of the Observer: On vision and Modernity in the Nineteenth century, Cambridge, Massachussetts, MIT Press, 1990 [L'Art de l'observateur: vision et modernité au XIXe siècle, tr. F. Maurin, Nîmes, Jacqueline Chambon, 1998], chap. II ("La camera obscura et son sujet»). Martin Jay fournit également des commentaires utiles et des informations bibliographiques sur le modèle de la camera obscura et sa relation à la philosophie française dans Downcast Eyes: The Denigration of Vision in Twentieth-Century French Thought, Berkeley, University of California Press, 1994. Plus spécifiquement, pour une discussion sur la question de l'abstraction dans le domaine de l'optique chez Descartes, voir D. Judovitz, «Vision, Representation, and Technology in Descartes ", dans D. Michael Levin (dir.), Modernity and the Hegemony of Vision, Berkeley, University of California Press, 1994. 
Bien que ce modèle abstrait hérité de Descartes ait conservé une forte influence sur la conception du sujet voyant, il a peu à peu été supplanté au $\mathrm{XIX}^{\mathrm{e}}$ siècle, en raison de l'intérêt croissant porté à l'observation expérimentale des images rétiniennes effectives. Dans Optique physiologique (1867), Hermann von Helmholtz explique comment les images rétiniennes sont observées avant 1851. Il commence par l'analogie classique:

L'oil se comporte essentiellement comme une chambre noire [...]. On peut la rendre visible sur des yeux fraîchement extraits, en enlevant avec précaution la partie centrale postérieure de la sclérotique et de la choroïde, laissant la rétine intacte et tournant vers des objets éclairés la cornée d'un œil ainsi préparé. On voit alors se dessiner sur la rétine de cet œil une image petite, lumineuse, nette et renversée. L'image est encore plus nette si, à l'exemple de Gerling, on enlève les éléments de la rétine au moyen d'un pinceau, et qu'on place ensuite dans l'ouverture une lamelle de verre ou de mica ${ }^{8}$.

Comme l'explique Helmholtz, il a été nécessaire de découper préalablement la membrane opaque qui fait obstacle à l'observation directe de la surface rétinienne. Qu'elle soit effectuée à partir d'yeux "fraîchement extraits " sur des moutons ou des bœufs, ou, comme en 1625, à partir d'un œil humain prélevé sur un cadavre, l'observation des images rétiniennes requiert l'élimination de l'opacité naturelle de cette membrane. Quoique ces expériences aient eu pour objectif d'élucider le fonctionnement de la vue chez l'homme, les scientifiques se sont tournés vers une autre espèce, dont l'anatomie optique semblait résoudre le problème de l'opacité. Helmholtz de poursuivre:

Il est également assez facile de voir les images rétiniennes dans les yeux des lapins blancs, dont la chorö̈de manque de pigment. Chez ces derniers, il n'est pas même nécessaire d'enlever la sclérotique: on voit l'image à travers cette membrane; elle est assurément moins nette que sur la rétine mise à nu, mais elle est suffisamment distincte pour qu'on puisse en reconnaître la position.

L'œil du lapin constituait le choix rêvé pour étudier la vue, parce que son déficit pigmentaire approchait l'idéal cartésien de transparence sur lequel reposait le modèle de la camera obscura. Et pourtant, il n'est pas tout à fait transparent. Comme le reconnaît Helmholtz, l'observation de l'image rétinienne dans l'œil d'un lapin n'est pas absolument claire et distincte. Ni totalement transparent ni totalement opaque, cet objet expérimental que constitue la rétine du lapin fragilise bien plus qu'il ne confirme le modèle cartésien de la vue. Au cours des XVIII ${ }^{\mathrm{e}}$ et XIX ${ }^{\mathrm{e}}$ siècles, les études consacrées aux phénomènes subjectifs de la vision ont conduit à un affaiblissement du modèle dominant de la camera

8. H. von Helmholz, Optique physiologique, trad. E. Javal, L'Harmattan, 2009, t. I, p. 86. 
obscura, relayé par une appréhension plus corporelle et physiologique de la perception visuelle. L'ophtalmoscope, inventé par Helmholtz en 1851, permettait d'observer les images rétiniennes sur l'œil humain - si bien qu'il n'était désormais plus nécessaire pour essayer de comprendre la vue d'aller chercher des modèles mathématiques abstraits ou des yeux d'animaux qui ne correspondaient qu'approximativement à ceux des hommes. Outil heuristique permettant la compréhension de la vue chez l'homme, l'œil de lapin occupe donc un espace intermédiaire dans l'histoire de la science entre l'objectivité transparente des modèles mécanistes et la subjectivité incarnée, localisée et vivante de l'œil humain.

C'est ainsi qu'il oscille entre deux conceptions de la vue: entre transparence et opacité, entre abstraction et anatomie, entre observation dépersonnalisée et perception centrée sur le sujet. C'est en gardant cette idée à l'esprit qu'on se propose de lire Triptyque (1973), un roman expérimental dont on peut considérer qu'il se compose presque exclusivement d'images rétiniennes, et dans lequel l'œil tranché d'un lapin fait vaciller deux modèles de représentation de la vue.

\section{TRIPTYQUE}

Dans un contexte marqué par l'influence des théories structuralistes de Jean Ricardou et les attaques frontales de celui-ci contre l' "illusion référentielle », Claude Simon a écrit Orion aveugle (1970), Les Corps conducteurs (1971), Triptyque (1973) et Leçon de choses (1975), avec l'objectif avoué de purger les "mauvaises habitudes inculquées " de la représentation mimétique9. La référentialité psychologique des œuvres antérieures s'est vue remplacée par une incohérence fragmentaire, ludique et chargée de jeux langagiers ${ }^{10}$; avec Les Corps conducteurs (texte retravaillé et augmenté à partir d'Orion aveugle), Simon s'est lancé dans une quête de la pure productivité du texte, considéré dans sa réflexivité et son auto-engendrement. Pourtant, il reconnaît lui-même qu'il n'a pas atteint son objectif anti-mimétique. Quoique relevant d'un dis-

9. Voir la préface à Orion aveugle.

10. Dans la remarquable étude qu'elle consacre à la visualité dans l'œuvre de Simon, Celia Britton constate que "depuis l'introduction de "O" au cour de La Bataille de Pharsale jusqu'à Leçon de choses, en passant par Les Corps conducteurs et Triptyque, on observe un mouvement de génération textuelle toujours plus libre, et toujours moins ancré dans la perception, le souvenir ou l'imagination par un sujet psychologiquement cohérent d'un monde diégétiquement cohérent " (Writing the Visible, Cambridge, Cambridge University Press, 1987, p. 15-16). 
positif langagier complexe, le roman n'en est pas moins toujours réductible à l'expérience d'un sujet humain: de même que les scènes d'Histoire peuvent être lues comme une succession de souvenirs et d'expériences fait(e)s par un individu, celles des Corps conducteurs peuvent s'interpréter comme l'inventaire non chronologique des hallucinations et des souvenirs d'un homme malade. Simon a beau avoir éliminé la narration à la première personne, il n'en a pas moins conservé la possibilité d'une lecture psychologique et phénoménologique ${ }^{11}$. David Carroll explique cet "échec" par une forme qui n'est pas " assez pure, plurielle ou discohérente pour supprimer toute possibilité d'assignation à une origine extérieure et d'explication en termes de références à une entité "non linguistique" ou "extratextuelle" 12 ".

L'échec théorique des Corps Conducteurs a poussé Simon à écrire Triptyque, roman qu'il considère comme une réussite formelle, en raison de l'abolition complète de toute référence extratextuelle.

J'avais le projet de faire un roman irréductible à tout schéma réaliste, c'est-à-dire un roman où les rapports entre les différentes " séries " (ou " ensembles ») ne relèveraient pas d'un quelconque enchaînement ou déterminisme d'ordre psychologique, ou encore de similitudes de situations ou de thèmes [...], et où encore il n'y aurait pas de personnages, de temps ou de lieux apparemment privilégiés, ce qui avait permis à certains critiques de résumer Les Corps Conducteurs en disant: un homme malade marche dans une rue et se souvient ${ }^{13} \ldots$

La charge de Simon contre le réalisme vise en particulier la subjectivité psychologique, l'univocité de perspective et la possibilité pour l'œuvre d'être résumée. Sa stratégie pour Triptyque repose sur le procédé scriptural de démultiplication des mises en abyme. Comme dans Histoire et Orion aveugle, les représentations picturales (cartes postales, photos) ont une fonction génératrice dans les descriptions du roman, mais, dans le cas de Triptyque, les imbrications référentielles des différentes scènes, multipliées à l'infini, créent un espace vertigineux qui défie la logique représentationnelle. Pour Lucien Dällenbach, la structure tripolaire de Triptyque réalise « la fin des illusions totalisantes " par son refus d'établir une relation hiérarchique entre

11. Comme le remarque Jean Duffy, l'expérience de la maladie (une tuberculose l'a cloué au lit pendant cinq mois) a exacerbé chez Simon la conscience de sa sensibilité perceptive et de la perméabilité de son corps aux influences extérieures ("Claude Simon, Merleau-Ponty and perception ", French Studies, $\mathrm{n}^{\circ} 46,1,1992$, p. 33-52, en particulier p. 36).

12. D. Carroll, The Subject in Question: The Language of Theory and the Strategies of Fiction, Chicago, University of Chicago Press, 1982, p. 188.

13. J. Ricardou (dir.), Claude Simon: Analyse, théorie, UGE, 1975, p. 424, cité dans L. Dällenbach, Le Récit spéculaire: essai sur la mise en abyme, Le Seuil, 1977, p. 193. 
le contenant et le contenu, entre l'image et le cadre, entre la représentation et le référent ${ }^{14}$. Mais ce principe d'une « multiplicité non hiérarchisée » dans Triptyque a été contestée par ceux qui relèvent la prédominance dans le roman des scènes de la série rurale, une prédominance qui sape théoriquement - ou à tout le moins déséquilibre - la triple structure intra-référentielle du roman ${ }^{15}$. Nous nous proposons par conséquent de revenir sur cette question de la non-référentialité de Triptyque en examinant de quelle manière la narration fait l'objet d'une dé-subjectivation du regard.

L'une des composantes essentielles de l'apparente auto-référentialité de l'œuvre réside dans l'élimination de la perspective narrative. Non seulement il est impossible de subsumer aucune des trois séries aux deux autres, mais il est impossible d'identifier quelque entité humaine que ce soit dont la perception serait à l'origine de ces scènes. Aucun des personnages qui peuplent Triptyque - les jeunes garçons et jeunes filles de la campagne, l'actrice et son amant, les spectateurs au cinéma, la jeune mariée et l'époux adultère, etc. - n'émerge en tant que conscience filtrante ou comme source de l'énonciation. Relevant d'observations purement constatives et neutres qui ne sauraient être rattachées à l'expérience d'un sujet, le roman résiste à toute tentative de classification au sein des catégories traditionnelles du point de vue.

Il n'en demeure pas moins que la fonction visuelle de l'observation en elle-même est incarnée dans le texte par la présence d'un sujet grammatical a-personnel, " on ». On ne saurait lire Triptyque sans remarquer la récurrence d'expressions telles que " on voit ", " on peut voir » ou " on perçoit " pour introduire les diverses descriptions du roman. Dans la mesure où le temps utilisé pour ces descriptions est toujours le présent, on pourrait être tenté d'avoir une lecture référentielle du roman et de l'interpréter comme la représentation réaliste d'un présent de perception phénoménologique. Après tout, Merleau-Ponty lui-même suggère que le « on » impersonnel saisit l'expérience perceptive avec plus de précision que le sujet à la première personne « je »: «si je voulais traduire exactement l'expérience perceptive,

14. Ibid., p. 193. Voir également L. Dällenbach, "Mise en abyme et redoublement spéculaire chez Claude Simon » [J. Ricardou (dir.), op. cit., p. 151-171], dans lequel Dällenbach considère Triptyque comme l'étape ultime de l'œuvre simonienne, laquelle s'est vue passer de l'unité centrée à la pluralité décentrée, de la représentation à la production, du reflet statique à la générativité.

15. Dans le cadre de l'une des discussions lors du colloque de Cerisy consacré à Simon, Christiane Mackward observe que la séquence rurale occupe davantage d'espace que les deux autres (maritime et urbaine), à quoi Simon répond que cette prédominance n'était pas intentionnelle (op. cit., p. 181-184). 
je devrais dire qu'on perçoit en moi et non pas que je perçois ${ }^{16}$ ». MerleauPonty évoque là une étape perceptive qui précède la constitution du Moi, mais il considère toujours que la perception se produit " en moi ». Simon, lui, va encore plus loin dans la dépersonnalisation de la perception, en retranchant du texte la personne par la conscience de laquelle les scènes pourraient être perçues. Faisant référence à l'emploi par Simon de l'expression " on voit » dans Triptyque et Leçon de choses, Celia Britton note:

Cette réduction du sujet-dans-la-langue au profit d'un primat apparemment absolu de la vue ne saurait en aucun cas s'analyser comme le retour au sujet perceptif quasi-phénoménologique des romans antérieurs, du fait de l'extrême dislocation du niveau de représentation dans ces textes, et de la prééminence de principes formels d'organisation. [...] Ainsi, la vision se voit transformée par la disparition virtuelle de la dimension référentielle: il ne s'agit plus d'un acte psychologique faisant l'objet d'une représentation, mais d'un dispositif textuel ${ }^{17}$.

Par l'emploi de l'impersonnel « on » qui occupe la place d'un sujet humain percevant, Triptyque rend abstraite la notion même de vision.

Car Triptyque est, avant tout, un roman de la vue. Certes, d'autres sens sont parfois convoqués ("On peut entendre le bruit tout proche de l'eau basculant par-dessus la murette d'un canal de retenue ", T, p. 744), mais le régime descriptif dominant dans Triptyque relève de la vue, y compris dans ces scènes qui semblent sortir du cadre statique de la représentation picturale, et qui sont évoquées telles qu'elles peuvent apparaître à un regard observateur: «De la grange on peut voir le clocher. Du pied de la cascade on peut aussi voir le clocher mais pas la grange. Du haut de la cascade on peut voir à la fois le clocher et le toit de la grange " (ibid.).

Des changements de perspective sont couplés à des variations de focalisation, par exemple lorsque tour à tour des fleurs et un clocher apparaissent nets ou flous, selon que la mise au point s'effectue sur le premier ou l'arrière-plan. Simon cite lui-même ce passage comme preuve que «ces images résultent du réglage de la lentille d'un objectif »; l'effet de totalité close de Triptyque passe selon lui par un point de vue déshumanisé et dépersonnalisé ${ }^{18}$. Il ne fait aucun doute que les descriptions multifocales du roman sapent toute possibilité de regard - ou survol ${ }^{*}$ - surplombant ou perspectif comme on le rencontre dans la narration omnisciente traditionnelle.

16. M. Merleau-Ponty, Phénoménologie de la perception, Gallimard, 1976 [1945], p. 249, cité dans J. Duffy, art. cité, p. 37.

17. C. Britton, op. cit., p. 66.

18. J. Ricardou (dir.), op. cit., p. 184. 
Dans Triptyque, le champ perceptif est constamment associé à la restriction, comme le montre dans la série campagnarde ce trou situé dans la paroi d'une grange qui permet d'entrevoir furtivement de la chair nue:

À l'intérieur de la grange, dans la pénombre, l'œil d'abord aveugle commence peu à peu à distinguer des formes mouvantes, l'éclat d'une chair blanche tranchant sur du noir. Les deux côtés rapprochés de la fente limitent étroitement, à droite et à gauche, le champ de vision. ( $T$, p. 748)

Réaction physiologique naturelle, l'œil - dont on ignore pour l'instant à qui il appartient (la suite permettra d'identifier deux jeunes garçons en voyeurs ${ }^{*}$ de basse-cour) - est momentanément aveuglé lorsqu'il passe d'un champ de vision très lumineux à l'obscurité d'une pièce fermée. Cette réaction, abondamment décrite dans les traités d'optique physiologique, est à classer parmi une liste de troubles visuels apparents qui - comme les points aveugles et les images différées - entament sensiblement le crédit de l'œil en tant qu'instrument fiable d'une vision claire ${ }^{19}$. En faisant explicitement référence à un champ de vision limité par les bords étroits d'un interstice, la description de la scène de la grange souligne les facultés limitées de l'œil (" [1] es deux côtés rapprochés de la fente limitent étroitement, à droite et à gauche, le champ de vision »). Un point de vue mobile et omniscient aurait poursuivi en décrivant directement ce qui se passe à l'intérieur de la grange; Simon, lui, organise sa description autour d'un point focal localisé, avec une particularité optique qui sape la maîtrise perspectiviste du point de vue traditionnel.

En fait, les références de Triptyque à la vue considérée comme phénomène physiologique marquent continuellement une rupture avec la conception optimiste d'une transparence de la vision, à l'origine des théories mimétiques de la représentation. Soit cette évocation d'une truite nageant dans une rivière:

19. Gillian Beer relève à la fin du $\mathrm{xIx}^{\mathrm{e}}$ siècle un questionnement philosophique concernant "l'authenticité de la vision de l'œil », en s'appuyant sur les résultats d'expériences menées en optique physiologique: "D'après les travaux de Helmholtz, l'œil lui-même se révèle un instrument imparfait, incapable de résolution stable " "Authentic Tidings of Invisible Things: Vision and the Invisible in the Later Nineteenth Century ", dans Teresa Brennan et Martin Jay (dir.), Vision in Context, New York, Routledge, 1996, p. 85). Martin Jay fournit une explication plus approfondie $\mathrm{du}$ lien entre physiologie et les critiques plus larges au $\mathrm{xx}^{\mathrm{e}}$ siècle de la maîtrise visuelle: "Si la science est consciente des capacités de l'œil, elle en connaît aussi les limites. L'œil humain perçoit des ondes lumineuses qui ne correspondent qu’à une partie du spectre total [...]. En outre, il entre dans sa composition un point aveugle, qui correspond à l'endroit où le nerf optique est relié à la rétine. On n'y prête pas attention parce que ce déficit est compensé par l'autre œil. Toujours est-il que le point aveugle suggère l'existence d'un "trou » métaphorique dans la vision, ce que [...] les détracteurs de l'oculocentrisme ont allègrement exploité » (op. cit., p. 8). 
la truite file comme une flèche et disparaît en amont dans la zone qu'occulte le reflet aveuglant du ciel, laissant persister sur la rétine la trace de sa forme allongée, rigide, simplement propulsée par les frétillements rapides de sa queue. ( $T$, p. 750)

Bien qu'il ne soit pas fait mention d'un sujet à l'origine de cette perception, la description présuppose l'existence d'un œil dont la relation et la réaction à la scène sont physiologiquement circonscrites. Comme pour la "fente* " de la grange, Simon évoque une faille dans le champ de vision - en l'occurrence le point aveugle causé sous l'eau par un reflet de lumière à la surface de l'eau ("la zone qu'occulte le reflet aveuglant du ciel »). Le poisson n'est plus visible quand il pénètre dans ce secteur. De plus, l'interaction de la lumière et de la forme sombre du poisson provoque un deuxième " point aveugle ", cette fois sur la rétine de l'œil qui perçoit: le point aveugle d'une image différée ${ }^{20}$. La perception d'un objet lumineux crée une image rétinienne qui persiste alors même que l'objet n'est plus là. La persistance de l'image fait obstacle dans un second temps à la perception normale du monde extérieur, le nouveau champ de vision se trouvant amputé par une sorte d'écran temporaire ou de "point aveugle" en forme de poisson ${ }^{21}$. Dans Triptyque, on trouve dans la description de la truite et de la rivière deux "zones occultées »: celle qu' " occulte le reflet aveuglant du ciel », et celle de l'œil percevant sur la rétine duquel s'est imprimée l'image différée de la truite. Dans ces lignes, la lumière fait entrer cet œil percevant dans une relation de spécularité réciproque et aveuglante - bien loin de la visée traditionnellement éclairante de la lumière, métaphoriquement garante de la connaissance. Par cet effet de miroir réciproque, Simon fait vaciller le primat du sujet voyant sur la réalité perçue. Il crée un univers textuel dans lequel l'œil et l'objet existent au même niveau de représentation (tous deux mas-

20. Comme les chercheurs en optique physiologique l'ont expliqué, les images différées - ou « impressions lumineuses persistantes "- affectent momentanément un certain nombre de cellules rétiniennes, ce qui affecte la capacité de l'observateur à percevoir les objets extérieurs du monde: "après l'action de la lumière sur la rétine, l'état d'excitation de l'appareil nerveux visuel persiste pendant quelque temps, et $[\ldots]$ cette persistance de l'impression est surtout facile à remarquer lorsque après avoir regardé des objets éclairés, on dirige l'œil sur un champ visuel tout à fait obscur. On trouve, en outre, qu'après l'action d'une lumière sur une portion quelconque de la rétine, cette portion modifiée perçoit la lumière incidente d'une autre manière que les parties de la rétine qui n'ont pas été affectées préalablement. Il s'agit donc aussi d'une modification apportée, par l'action de la lumière, dans la manière dont l'appareil nerveux visuel est sensible aux excitants extérieurs " (Helmholtz, op. cit., $\$ 23$ «Des modifications de l'excitabilité », p. 357).

21. On rappelle que l'effet d'image différée participe d'une défiance accrue dans les capacités de l'œil. La vision ne peut plus être considérée comme relation transparente entre le sujet voyant et le monde objectif quand la physionomie même de l'œil peut créer des objets de perception qui n'ont pas d'existence extérieure. 
qués par des points aveugles, l'un n'ayant pas plus de « réalité » que l'autre), de telle sorte que la physiologie optique se voit mise au service de la non-référentialité linguistique.

Les scènes de Triptyque sont vues. Les images du roman se fixent temporairement sur la rétine fictionnelle, l'une succédant à l'autre sans transition, comme dans ces dernières lignes d'Orion aveugle analysées plus haut: "la mince membrane de sa rétine sur laquelle les images du monde viennent se plaquer, glisser, l'une prenant la place de l'autre ». Et de même que la description anatomique de l'œil dans Orion aveugle, le point de vue dans Triptyque se situe à cheval entre ces deux extrémités que sont l'abstraction et la corporéité. D’un côté, la vue se voit réduite dans le roman à un œil mécanisé qui n'est rattachable à aucun observateur humain; de l'autre, la description du champ perceptif est soumise aux limites physiologiques de l'œil humain. Dès lors, Triptyque semble échapper aux conventions narratives issues des deux principales philosophies de la vision: a) la vision empiriste, enracinée dans l'expérience contingente d'un observateur incarné; et b) le perspectivisme cartésien, fondé sur la foi dans le mythe de la maîtrise visuelle et de la transparence.

Survenant dans les premiers temps de la "période structuraliste" de Simon, l'image picturale qui clôt Orion aveugle - un bout de muscle oculaire à moitié arraché du reste de la tête - pourrait bien être considérée comme l'expression d'une séparation progressive dans l'œuvre du romancier entre l'œil et l'être. Par l'utilisation de l'expression " on voit " qui sépare la perception visuelle de la subjectivité humaine, Triptyque se rapproche de l'objectif d'auto-référentialité qu'Orion aveugle et Les Corps conducteurs avaient échoué à atteindre, selon le dire même de Simon. Désormais délivré de tout lien avec un sujet sensible - corps et esprit - qui serait à l'origine des images du roman, l'œil dans Triptyque n'est plus qu'un dispositif visuel neutre, un " agent » impersonnel de perception. Simon refuse dans son roman de fonder la vision sur une philosophie du sujet, en invoquant les structures mêmes de la perception, contre la référence au sujet perceptif humain. La quête de la pure autoréférentialité dans Triptyque conduit à retrancher l'œil du sujet, ou encore, pour reprendre un jeu de mots bien connu, il dissocie l'œil du « je » 22 .

On ne sera donc pas surpris de trouver dans ce roman où les mises en abyme pullulent la description d'un œil en train d'être extrait d'un corps. Mais cette énucléation ne concerne pas un sujet humain, et la scène, en 
toute rigueur, ne fait pas partie de la triple structure spéculaire que Simon a organisée à dessein autour de séries de représentations picturales (cartes postales, gravures, extraits de films). Et pourtant, elle menace le dispositif anti-référentiel clos sur lui-même qui semblait être l'ambition de Simon pour Triptyque. Ce que nous nous proposons à présent de montrer.

\section{L'CEIL DU LAPIN (II)}

Parmi les nombreuses scènes campagnardes du roman, on rencontre le motif récurrent de la vieille femme qui prépare un lapin pour le cuisiner ${ }^{23}$. En plusieurs séquences dispersées et désordonnées, " on voit " cette vieille femme extraire le lapin de son clapier, l'attacher à une branche, le tuer avec une bûche, le saigner, l'écorcher et le poser sur la table de la cuisine. La saignée du lapin en particulier donne lieu à des détails très crus:

Armée de nouveau de son couteau dont la pointe de la lame dépasse seule de son poing fermé, la vieille à la tête de chien arrache d'un geste rapide du poignet l'un des yeux du lapin. En même temps sa main gauche tend au-dessous de l'orbite évidée un bol au bord ébréché. [...] Glissant vers le fond les gouttes de sang s'étirent en ovales, se font plus serrées, et bientôt un filet vertical relie l'orbite vide au bol où le niveau du sang recueilli s'élève peu à peu. ( $T$, p. 757-758)

Bien que l'image soit violente et sanglante, la vieille femme exécute le geste machinalement, comme si elle avait déjà accompli une semblable corvée à de nombreuses reprises. On pourrait être tenté de voir dans la séparation de l'œil du lapin d'avec son corps une image du projet plutôt mécaniste de Triptyque qui se propose de séparer la perception visuelle du sujet voyant. Plus encore, le vide laissé par l'énucléation du lapin, un vide entre l'œil mort et le corps vivant, pourrait être considéré comme une image de l'espace que Triptyque lui-même occupe entre deux modalités disjonctives de production narrative - l'une centrée autour d'un sujet incarné, et l'autre neutre et désincarnée.

L'orbite vidée du lapin semble n'être que l'une des nombreuses images de disjonction qui peuplent Triptyque. Mais cette image se rencontre dans les scènes de la série campagnarde qui, quand on les isole des deux autres séries, peuvent se résumer en une succession d'événements formant une intrigue

23. Pour un aperçu critique de la résonance intertextuelle du motif de la « vieille » chez Simon, voir R. Sarkonak, Les Trajets de l'écriture: Claude Simon, Toronto, Paratexte, 1994, p. 71-72 et 202. Voir également l'article désormais classique d'A. C. Pugh, "Du Tricheur à Triptyque, et inversement ", Études littéraires, vol. 9, $\mathrm{n}^{\circ} 1$, avril 1976, p. 137-160. Je tiens à remercier Ralph Sarkonak pour avoir attiré mon attention sur l'article de Pugh et, plus largement, pour ses précieuses suggestions et ses encouragements. 
linéaire - même s'il va de soi qu'une telle reconstitution aille à l'encontre de la visée formaliste du roman. Même s'ils ne sont jamais nommés, les différents personnages reparaissent suffisamment souvent pour que l'on puisse identifier les relations qui les unissent. Ainsi d'une domestique qui confie une petite fille dont elle a la garde à deux jeunes garçons qui pêchent dans la rivière, avant de s'éloigner en direction de la grange en compagnie d'un jeune chasseur. À un autre moment, les deux garçons confient à leur tour la petite fille à trois gamines à peine plus âgées, pour se diriger vers la grange où une fente dans la paroi leur permet d'observer les ébats de deux amants (on devine qu'il s'agit de la nounou et du chasseur). Dans une autre scène, les trois gamines plus âgées s'éloignent elles aussi, laissant la petite fille sans surveillance sur le bord de la rivière. C'est ainsi que peut être reconstituée une succession de péripéties par laquelle une petite fille passe de main en main. Bien sûr cette succession est entrecoupée de fragments de scènes provenant des deux autres séries, celles de l'estaminet et de la cité balnéaire. Quand la série campagnarde fait son retour, la petite fille est en train de cueillir des fleurs sur le bord de la rivière. On peut voir dans le long extrait qui suit comment le texte glisse sans transition d'une description très factuelle à une autre (en l'occurrence, de la petite fille sur le bord de la rivière à un numéro de clown avec singe):

La petite fille reste seule, sa robe faisant une tache claire sur le vert soutenu du pré. Avec des gestes maladroits elle arrange son bouquet, regarde autour d'elle, se penche sur le côté pour cueillir une fleur, [...] regarde de nouveau autour d'elle, se lève, puis, avisant une touffe de fleurs mauves à longues tiges qui poussent sur la berge, s'approche de la rivière. L'eau glisse rapidement avec un bruit soyeux, presque imperceptible, agitée de faibles remous le long des bords ou de tourbillons qui s'enroulent à sa surface et fuient, emportés par le courant. On distingue avec netteté le tuf jaunâtre et les cailloux qui tapissent le fond, les plus larges couverts par endroits d'une mousse noire. Une truite, noire aussi, traverse en oblique le courant, et disparaît sous le surplomb de la berge. Entre les feuillages sombres reflétés jouent des fragments de ciel, comme des flaques d'argent, ondulant à peine. En même temps qu'il précipite ses appels de langue et ses encouragements, le clown blanc imprime au manche de la chambrière de brèves secousses qui se transmettent comme une onde tout le long de la corde ( $T$, p. 863-864)

Aucun fait saillant dans cette évocation de la petite fille au bord de la rivière. L'enfant s'approche des fleurs qui poussent sur la berge, une truite nage sous l'eau, le ciel se reflète à la surface de la rivière; puis le lecteur est emporté vers les autres séries avec des descriptions minutieuses - le clown, une scène de sexe adultère très arrosée, etc. Lorsque le lecteur retrouve la série campagnarde, la petite fille n'est plus là. Mais il y a le lapin: 
Personne [...] ne semble s'être soucié du lapin dépouillé dont les pattes aux bottillons de fourrure et la tête sanglante dépassent du torchon au liséré rose [...]. C’est la nuit. L'ampoule de faible puissance qui éclaire la cuisine projette sur la toile cirée qui recouvre la table l'ombre noire de la tête à l'orbite vide et celles des deux pattes raides et parallèles, à demi pliées au-dessus de la tête dans un geste d'ultime protestation. ( $T$, p. 868)

Avec sa tête et des pattes qui dépassent comme en signe de protestation (contre quoi? contre sa propre mort ou celle d'un autre?), le lapin, laissé à l'abandon dans son plat sans avoir été cuisiné alors qu'il fait nuit depuis longtemps, devient un indice pour le lecteur, le signe que quelque chose ne va pas dans cette scène domestique. À côté du lapin, la nounou est assise, elle est en pleurs et se couvre les yeux avec les plis de son tablier; et la vieille femme regarde droit devant elle, "[l]e regard voilé d'une taie blanchâtre " (p. 869). On entend des sanglots émanant de la chambre voisine. À ce moment-là, le lecteur marque une pause et se demande ce qui bien a pu se passer entre ces deux situations, l'enfant laissée sans surveillance qui se penche au-dessus de la rivière, et ce cadavre de lapin abandonné là sur la table - un questionnement qui n'a aucun sens dans une lecture non-mimétique du roman, en vertu de laquelle la seule réalité de Triptyque est celle d'un auto-engendrement textuel qui détermine la succession des séquences. L’organisation du roman ne saurait être déterminée par un référent extra-textuel; aucune histoire, aucune vérité ne sont à chercher " en dehors" ou "audelà » de la surface du texte. Certes. Et pourtant, cette tête borgne de lapin, décrite comme "dépassant " le rebord du plat et l'extrémité d'un torchon, ne peut qu'attirer notre attention sur un aspect qui "dépasse » la textualité pure à laquelle aspire le roman. Qu'y a-t-il au-delà de la surface du texte? La mort d'une petite fille.

Les lecteurs du roman, et Lucien Dällenbach avec eux, en ont conclu

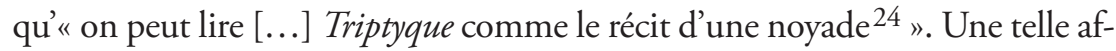
firmation a de quoi surprendre si l'on considère qu'il n'est jamais fait mention de quelque noyade que ce soit. Comment un texte qui se présente comme une pure production langagière, sans référent extra-textuel, peut-il se faire l'histoire de quelque chose qui ne s'y trouve pas, qui resterait en dehors des faits relatés? Non seulement l'analyse de Dällenbach ajoute au roman un épisode qui lui est extérieur, mais surtout le résumé du roman se voit centré autour de ce même épisode. Rappelons-nous qu'à la suite des Corps conducteurs, Simon s'était donné l'objectif - atteint, selon son propre jugement - d'écrire

24. L. Dällenbach, Claude Simon, op. cit., p. 41. 
un roman qu'il serait impossible de résumer. La visée anti-mimétique est sérieusement contestée par la possibilité de réduire Triptyque à la représentation d'un événement - la perte d'une vie humaine -, d'autant plus que l'événement en question se produit sur le plan référentiel d'un au-delà* du texte, que Robbe-Grillet et d'autres praticiens du Nouveau Roman vouaient aux gémonies.

On pourrait certes faire valoir que c'est seulement par la force d'une vieille habitude herméneutique qu'on s'acharne obstinément à lire Triptyque comme l'histoire d'une noyade. Ce serait ne pas voir que le texte lui-même fournit la clé d'une telle lecture, disséminant des indices tout au long de l'œuvre, depuis la description, tôt dans le roman, de la femme faisant l'amour, « [s]a chevelure détrempée pendant comme celle d'une noyée, ses yeux aveugles de noyée grands ouverts sur le noir » (p. 775), jusqu’à la scène finale de recherches avec lampes torches dans les bois, en passant par les multiples références aux "noyers 25 ». En théorie, aucune des trois séries de mises en abyme n'est censée dominer les autres, mais la disparition de l'enfant qui survient peu avant la fin du roman (localisation qui en constitue traditionnellement l'acmé), après avoir fait monter peu à peu le suspense par des abandons successifs, confere une certaine prééminence à la série campagnarde, certaines scènes des deux autres séries pouvant alors être réduites à des écrans visuels ou un terrain contenant des indices de ce qui manque. Parmi les indicateurs textuels qui autorisent le lecteur à imaginer un "au-delà " référentiel dans lequel une petite fille trouve la mort, il y a cette image du lapin écartelé « dans un geste d'ultime protestation ». Ce corps flasque et pathétique oriente notre attention vers la perte d'un autre corps. Et l'orbite vide du lapin signale une autre béance: le vide diégétique de la noyade. Arraché puis jeté à la poubelle, l'œil du lapin représente peut-être la vision humaniste qui a été retranchée des procédures fictionnelles du formalisme. Malgré toutes les occurrences de l'expression "on voit ", aucun oil n'aura été témoin de cette perte humaine que constitue la noyade d'un enfant.

L'extraction de l'œil du lapin n'a pas laissé un vide, une simple absence qui impliquerait un simple effacement du sujet. Au lieu de cela, elle laisse

25. Sur la dispersion (ou " dissémination ") sémantique de la "scène occultée de la noyade nocturne " dans Triptyque, voir S. Lotringer, "Cryptique », dans J. Ricardou (dir.), op. cit., p. 325. Celia Britton observe à juste titre que Lotringer a beau affirmer que Triptyque défie toute tentative de décryptage, la formule même qu'il emploie ("scène occultée de la noyade nocturne ") implique qu' " en cette occasion, au moins, le lecteur a la possibilité de retrouver un sens caché précis, et il ne manque pas de le faire" (op. cit., p. 134). 
derrière elle la trace d'une séparation violente sous la forme du sang qui s'écoule, goutte à goutte, de l'orbite vidée. Cette liquidité est à relier aux larmes de la domestique qui était chargée de surveiller la petite fille, ce qui permet de suggérer des sentiments de culpabilité, de nostalgie et de perte sur un ton paradoxalement neutre. Mária Minich Brewer met en garde contre la tentation de réduire la "violence sublimée » de Triptyque à une " analyse formelle" qui supprimerait l'émotion suscitée par la mort d'un enfant ${ }^{26}$. En analysant la violence contenue dans le roman au moyen d'un lexique psychologisant ("sublimation", "affect»), elle reconnaît implicitement que Triptyque relève de ce réalisme psychologique qui caractérise les romans antérieurs de Simon. On entend ici montrer quau sein de la diégèse de Triptyque, ce point aveugle, cette mort par noyade invisible et non figurée, constitue le vide qui rend possible le lien entre ce roman structuraliste et les œuvres précédentes de Simon. Les traces laissées par ce vide suggèrent la perte de quelque chose qui a été violemment arraché. Dans une certaine mesure, l'énucléation violente du lapin ne signale pas seulement la perte d'un corps humain, mais la nostalgie d'un certain type de récit où le sujet sensible est envisagé dans une perspective psychologique comme source d'images romanesques.

La dernière scène de la série campagnarde évoque les lumières de lampes torches disséminées parmi les bois dans les ténèbres de la nuit. On procède à une fouille des lieux pour retrouver le corps de la petite fille et les hommes se dispersent, tout juste équipés de cette extension mécanique de leur œil, la "petite lumière " de leurs " lampes de poche" (p. 871-873). Le ton dans cette séquence est sensiblement moins neutre que dans le reste du roman. Du chant des criquets et des palpitations à peine perceptibles sourd une atmosphère sinistre: "La terre, le monde tout entier, semblent engloutis sous une couche d'encre épaisse, palpable, dont on peut sentir le contact, comme liquide, sur les globes des yeux, le visage, les membres même 27 "(p. 871).

26. M. Minich Brewer, Claude Simon: Narrativities without Narrative, Lincoln, University of Nebraska Press, 1995, p. 104-106. Brewer introduit le concept de "narration parodique" pour désigner l'attitude de défense du texte simonien contre la perte, l'aliénation, la trahison et la mort.

27. J. H. Duffy souligne la dimension éminemment phénoménologique de ces lignes qui évoquent ce que la nuit empêche de voir et permet d'entendre. Suivant l'intérêt de Merleau-Ponty pour les conditions inhabituelles de perception qui permettent d'attirer l'attention vers ce qui se situe audelà du champ ordinaire de perception, elle conclut: "Ce passage ne relève pas d'une description animiste qui viserait à illustrer un principe de renouvellement perpétuel de la Nature par-delà la mort des individus: il vise avant tout à proposer un point de vue singulier sur le réel et à suggérer un foisonnement de choses tout autour de nous que nous ne remarquons pas " (art. cité, p. 46). 
Les lampes sont rendues inefficaces, "dérisoires au sein des ténèbres ", par la " couche d'encre » qui engloutit tout. On peut ainsi dire que l'œil perdu, l'œil arraché de son orbite, a été remplacé par des yeux aussi mécaniques que vains - signe, peut-être, que la logique narrative, au sein de laquelle la perception a été séparée du sujet, est elle-même " dérisoire ": stérile, mécanique, insuffisante.

Autrement dit, il semble bien que Triptyque intègre en son sein la critique même formulée par de nombreux lecteurs, à savoir qu'en tant qu'exercice purement formel et stérile, il est passé à côté de quelque chose. La situation du roman dans l'œuvre de Simon permet de soutenir ce point de vue, dans la mesure où après la période très expérimentale des années 1970, Simon est revenu à des formes romanesques plus conventionnelles: Les Géorgiques (1981) et L'Acacia (1989) s'organisent autour de thèmes humanistes fondamentaux tels que la guerre, la famille et l'histoire, tandis que Le Jardin des Plantes (1997) peut être lu dans une perspective autobiographique. Avant la publication des Géorgiques, Simon semblait déterminé à poursuivre dans la voie structuraliste, une voie qui liquide le narrateur doué de psychologie et le pathos humaniste qui va avec. Randy Birn analyse les manières successives de Simon comme l'alternance entre d'une part l'investigation des possibilités du langage à travers une "pratique radicale de la description auto-génératrice " et d'autre part "l'obsession des grandes questions existentielles: le temps, l'espace, la nature, la guerre, l'amour ${ }^{28}$ ". Ces thèmes humanistes ont conduit Morton Levitt à qualifier Simon de "survivant moderniste ". Tandis que des écrivains comme Robbe-Grillet ou Butor expérimentaient dans les années 1950 et 1960 des techniques radicalement post-humanistes, les préoccupations de Simon sont demeurées proches de celles de Proust, Joyce ou Faulkner ${ }^{29}$. Levitt affirme ensuite que même dans la période structuraliste des années 1970, les romans simoniens conservent le pathos des romans antérieurs: "Les préoccupations humanistes émergent d'une façon ou d'une autre, y compris dans un roman aussi stylisé et fabriqué que Triptyque. " Levitt n'analyse pas de quelle façon ces préoccupations se manifestent dans le roman, si ce n'est qu'il suggère que la mort de la petite fille suscite un sentiment de responsabilité et de culpabilité chez l'un des

28. " Postface ", dans R. Birn et K. Gould (dir.), Orion blinded: Essays on Claude Simon, Lewisburg Pa, Bucknell University Press, 1981, p. 289.

29. M. P. Levitt, "Modernist Survivor: The Later Fiction of Claude Simon ", Orion blinded, éd. cit., p. $260-284$. 
personnages de la série campagnarde, en l'occurrence l'un des jeunes garçons. Il poursuit: "Cela signifie-t-il que le garçon, l'un des rares centres de conscience de Triptyque, soit en quelque sorte relié à Georges [le narrateur alter ego de Simon]? Le fait même que la question se pose indique [...] que Simon n'a pas totalement renoncé à sa vieille technique, à sa propre histoire, à ses besoins et inquiétudes humanistes ${ }^{30}$. " Pour pouvoir formuler sa question, Levitt a besoin de tenir pour acquise la mort de la petite fille dans le roman. Il semble pourtant que la vraie question, celle qui sème le doute au sein du projet anti-humaniste et anti-représentationnel de Triptyque, c'est la question à laquelle répond la noyade de la petite fille: "Que s'est-il passé? " ou «Qu'est-ce que le texte passe sous silence?" En effet, c'est dans ce trou diégétique - ou, plus précisément, dans ce point aveugle, compte tenu de la récurrence structurante de l'expression " on voit»-que le pathos et le sentiment d'une perte humaine réinvestissent le texte simonien.

Quand, avec Les Géorgiques et L'Acacia, Simon revient à un mode de représentation humaniste, il délaisse le " on voit " impersonnel pour élargir le spectre du point de vue à des perceptions, des sensations et des remémorations subjectives. Dans les années 1970, Simon, comme Robbe-Grillet, a privilégié de manière radicale la perception visuelle, considérée comme moyen de mettre en question le sujet conçu comme point d'ancrage de la signification romanesque. Pourtant, déjà dans Triptyque, l'œil désincarné du lapin marque une ambivalence narrative à l'égard du sujet désincarné de l'expérience formaliste. Les remarques de Helmholtz citées en début d'article rappellent comment la recherche en optique physiologique utilisait l'œil de lapin pour tenter de comprendre la vision humaine dans une perspective objectiviste et a-corporelle. L'opacité partielle de cet œil le reliait pourtant à cette subjectivité corporelle à laquelle il était censé échapper. De même, dans Triptyque, l'œil énucléé du lapin évoque une textualité séparée de la subjectivité humaine, ses traces restent comme le signe d'une nostalgie opaque du corps perdu, un corps à situer dans l'écoulement du temps et la mort. Symbole d'une absence diégétique (la noyade jamais racontée d'une petite fille), l'œil du lapin en vient à manifester à la fois une écriture de type déshumanisé et non subjectif, et la nostalgie de ce que rejette cette écriture - l'être sensible. 


\section{PUNCTUM CAECUM}

Jetons un dernier regard sur le point aveugle de Triptyque, la scène absente de la noyade. Quoiqu'absente de la chaîne d'images descriptives, cette noyade* se manifeste au lecteur sous la forme de traces dispersées à travers le roman. Elle fonctionne ainsi comme un vide productif, un trou dans la chaîne de signifiants qui en tant que tel crée sa propre signification. Évoquant un autre topos simonien - celui d'un pan de rideau qui masque une femme désirable - Celia Britton propose une analyse qui nous paraît tout aussi bien convenir pour caractériser ce vide structurel de Tripyque: "la manifestation [...] d'une censure linguistique, donc d'une répression textuelle en quelque sorte, créant sur le plan de la représentation du discours des failles dans lesquelles peuvent alors s'immiscer des modalités plus libres de jeux langagiers associatifs - autrement dit elles opèrent en tant que lacunes génératives ${ }^{31}$ ». Britton établit un lien entre le principe de «lacune générative " et le concept lacanien de forclusion * Celui-ci correspond à un mécanisme de déni prenant la forme d'une faille ou d'un trou dans la conscience. Ce qui a été rejeté reparaît dans le champ du réel sous la forme d'hallucinations psychotiques ${ }^{32}$. Ce mécanisme est proche des concepts freudiens de refoulement ${ }^{*}$ et de retour du refoulé, mais Lacan va plus loin en affirmant que ce qui fait l'objet d'un " rejet " peut très bien ne pas avoir originellement existé. Lacan illustre la différence entre la forclusion* et le refoulement ${ }^{*}$ freudien par l'image du trou dans un tissu:

Si nous imaginons l'expérience comme une pièce d'étoffe constituée de fils entrecroisés, nous pourrions dire que le refoulement y serait figuré par quelque accroc ou déchirure, toujours reprisable, alors que la forclusion y serait figurée par quelque béance due au tissu lui-même, bref un trou original ${ }^{33}$.

Dès lors, la forclusion relève d'une faille inhérente à la texture même du tissu, un « trou originel » qui ne peut se manifester qu'à la surface de l'étoffe plutôt qu'en profondeur. (Une pièce prélevée d'un tissu peut continuer à exister, alors qu'un "trou d'origine " ne laisse aucune trace matérielle.) Transférons cette image textile du champ psychanalytique vers le champ littéraire: on peut alors voir en Triptyque un roman avec " trou d'origine", qui ne se manifeste qu'à la surface du roman. En tant que projet structu-

31. Op. cit., p. 163.

32. Voir M. Jay, op. cit., p. 354-356.

33. A. Lemaire, Jacques Lacan, Bruxelles, Mardaga, 1977, p. 341, citée dans C. Britton, op. cit., p. 223. 
raliste, Triptyque se présente comme une chaîne de signifiants sans signifié. À l'image du mécanisme de forclusion qui abolit les différences entre le Réel et le Symbolique, le roman structuraliste abolit la distinction entre signifiant et signifié. De sorte que l'image d'une petite fille qui se noie (ou encore l'affirmation: "une petite fille s'est noyée ») n'existe pas dans un au-delà du texte, pas plus qu'elle n'apparaît dans la " trame " du roman, mais son absence même se manifeste à la surface du texte.

Une image peut-être plus évocatrice de la faille narrative de Triptyque est à chercher dans un concept qui, au sein de la pensée lacanienne, précède la forclusion: la scotomisation. C'est Charcot qui dans les années 1880 l'emprunte à l'ophtalmologie. Elle désigne concrètement « une lésion rétinienne produisant un point aveugle ${ }^{34}$ ». L'analogie entre le point aveugle ophtalmologique et la «faille " ou le " trou " psychique fabriqués par le déni conduit Lacan à employer les termes de scotomisation et de forclusion dans des sens similaires ${ }^{35}$. Bien que ces notions renvoient toutes deux à des mécanismes de déni et de retour, celle de scotomisation entretient toutefois un lien plus direct avec la physiologie oculaire. Composé à partir d'images rétiniennes, Triptyque est un roman dans lequel la scène jamais narrée de la noyade opère comme une lésion rétinienne, un vide significatif à la surface du texte - comme la «taie blanchâtre" voilant le regard de la vieille femme qui tue le lapin ${ }^{36}$ (p. 869). Le regard y est toujours contrarié par une interférence, une perturbation, une restriction ou même un trou dans le champ de vision.

Tout au long du roman, le motif de l'œil est associé à l'idée de vide ou de trou. On pense bien sûr au "trou laissé par l'œil arraché [du lapin]" (p. 764), mais il y en a d'autres, moins explicites. Le roman fait à plusieurs reprises référence aux jeunes garçons qui regardent avidement des amants à l'intérieur de la grange à travers la fente de la paroi, "l'œil collé à la déchirure" (ibid.). On trouve même une fleur - « un œillet blanc tombé de la boutonnière de l'homme" (p. 773) - pour accomplir le passage de l'œil à

34. M. Jay, op. cit., p. 353-354.

35. «La notion de scotomisation [...] suggère qu'une tache aveugle réelle se produit quand quelque chose est ressenti comme trop menaçant pour être vu. Celle de forclusion va plus loin en considérant que la "vue" de la castration apparente de la mère déclenche une exclusion de l'image à rejeter, ou plus précisément, elle suscite une incapacité à l'intégrer sur un plan symbolique et verbal, provoquant sa réapparition dans le monde réel sous la forme d'hallucinations auditives (on entend des voix) ou visuelles " (M. Jay, op. cit., p. 356).

36. Aucun blanc typographique ne vient signaler ce trou dans le récit, contrairement à celui qui correspond au meurtre de la jeune fille dans Le Voyeur de Robbe-Grillet. La "trame " de Triptyque est ainsi conçue que l'absence d'un morceau du tissu ne se remarque pas immédiatement. 
l'œillet au sens de petit trou: celui de la boutonnière dans le tissu ${ }^{37}$. Au fil d'une autre séquence visuellement dense, on passe de l'évocation d'un lapin avec son " orbite vide » à celle d'un garçon faisant son devoir de géométrie, puis aux yeux des deux garçons regardant dans la grange à travers le trou de la paroi (p. 764). C'est par une logique d'ordre visuel que l'on peut tisser un lien entre la scène de devoirs, l'orbite vide du lapin et le regard avide des jeunes voyeurs ${ }^{*}$. En effet, le garçon déconcentré délaisse son devoir de géométrie et sort une loupe ainsi qu'une feuille de papier-calque:

Au centre du disque grisâtre projeté par l'ombre de la loupe sur la feuille de papier de soie [...] apparaît un rond minuscule où se concentrent les rayons de soleil qui frappent la surface de la lentille. En dépit de l'attention du garçon, la main qui tient la loupe est agitée de légers mouvements auxquels correspondent sur le papier d'infimes déplacements du foyer lumineux que le garçon s'efforce de maintenir au même endroit. Au bout d'un moment, le papier commence à roussir, puis, tandis que s'élève une légère fumée, un trou se forme, aux bords noircis qui vont s'élargissant. (ibid.)

Avec quelque maladresse en raison de tremblements mal maîtrisés, le garçon tente de concentrer les rayons lumineux sur le papier dont la combustion forme un trou. Une telle expérience optique est on ne peut plus anti-cartésienne: on est littéralement détourné de la géométrie (le devoir que le garçon est censé faire), le corps fait obstacle à la réalisation du projet, la lumière est contrainte de composer avec l'ombre ("l'ombre de la loupe»), et tout cela dans le but de créer du vide plutôt que d'édifier un savoir.

On peut associer ce trou issu de la combustion à la faille diégétique de la noyade sur la base non seulement d'un vague écho métaphorique, mais aussi d'une chaîne de culpabilité et de refoulement. Soient les lignes qui suivent l'extrait précédent:

Entendant un nouveau bruit à l'intérieur de la maison, le garçon range précipitamment la loupe et la feuille de papier de soie dans le tiroir de sa table qu'il repousse brusquement et reprenant son porte-plume il se penche au-dessus de la figure géométrique sur laquelle, sans cesser de prêter l'oreille au bruit, il trace sans conviction une autre droite tangente au cercle circonscrit à partir du sommet $\mathrm{B}$ du triangle initial. (ibid.)

L'utilisation de la lumière pour former un trou par combustion est suivie de la dissimulation précipitée des pièces à conviction. Cette attitude entre en résonance avec d'autres séquences romanesques associant activités illicites et

37. Dans son analyse de la distribution des termes significatifs de Triptyque, Guy Neumann inventorie une douzaine d'autres passages où "l'œil et la lumière " sont sémantiquement associés " au trou, au vide et au creux " (G. Neumann, Échos et correspondances dans Triptyque et Leçon de choses de Claude Simon, Lausanne, L'Âge d'homme, 1983, p. 123-124). 
culpabilité. Dans Triptyque, les yeux et les trous s'entrelacent pour former un réseau du savoir interdit. Un jeune garçon par exemple en interroge un autre, plus âgé, lui demandant sans arrêt à propos des amants de la grange: "Tu les as vus? ", à quoi l'autre répond: "Tu parles j'ai même vu dedans y a un trou » (p. 879), réponse aux accents très freudiens, puisque par un glissement de la référence, le "trou " passe de la paroi au vagin. Le voyeurisme est en outre lié à la mort de la petite fille dans la mesure où c'est en allant regarder par un trou dans la grange que les garçons laissent l'enfant seule au bord de la rivière. Par ces associations, lumière et vision deviennent constitutives d'un réseau tourmenté de mécanismes psychiques. Loin d'être garante du savoir scientifique positiviste, la lumière est associée dans Triptyque à ce même rôle d'écran qui s'observe dans la vision ou la psyché chez Merleau-Ponty, Freud ou Lacan quand ils développent leur théorie de la vision.

Tout au long du roman, la lumière fait obstacle à la vision au lieu de la faciliter. On y voit des rayons lumineux - qu'ils soient naturels ou artificiels - affecter et modifier l'œil avec force détails anatomiques. Un homme se tient debout, les yeux " mi-clos comme éblouis par la lumière du plafonnier " puis il cligne des paupières après avoir contemplé le ciel nuageux " pour protéger ses prunelles aveuglées" (p. 803-804); la mer scintille de reflets "sous le soleil de mille étoiles aveuglantes" (p. 833); les mots d'un message publicitaire lumineux qui défile " traîn[ent] derrière eux sur la rétine éblouie des crinières d'aigrette lumineuse» (p. 761); au cinéma, les yeux des spectateurs sont "éblouis par les lumières" (p. 865), et la combustion de la pellicule crée un trou qui donne à voir sur l'écran une " tache blanche, aveuglante" (p. 864), tissant par là un lien avec l'image évoquée plus tôt du papier brûlé grâce à une loupe. Littéralement, la lumière fait des trous - sur un écran de cinéma, sur une page blanche, sur la rétine. L'exemple le plus parlant de cette " opacité lumineuse " se trouve sans doute dans ces lignes que l'on se propose de citer à nouveau où la surface réfléchissante de la rivière se combine à l'image rémanente d'une silhouette de truite pour produire un point aveugle, redoublé et spéculaire:

la truite file comme une flèche et disparait en amont dans la zone qu'occulte le reflet aveuglant du ciel, laissant persister sur la rétine la trace de sa forme allongée, rigide (p. 750)

Le soleil aveuglant a partiellement occulté la surface de la rivière, la rendant opaque pour l'œil du spectateur. Dans le même temps, le soleil a aussi pratiqué un trou dans la rétine, créant une image différée ayant la forme du 
poisson ${ }^{38}$. L'image différée persiste sur la rétine, alors même que le poisson n'est plus là. Le phénomène optique d'image différée rend problématique la distinction entre réalité objective et perception subjective. La lumière provoque des points aveugles, mais ces points ne restent pas inertes. Ce sont des images-traces persistantes qui font écran au champ de vision premier. C'est cette double action - élimination par la lumière d'une portion de la rétine puis auto-génération d'une image - qui fonde la possibilité d'une analogie entre la structure de Triptyque et le concept lacanien de scotomisation. D'une certaine façon, la " lacune générative » que constitue la noyade de la petite fille se trouve déjà inscrite dans la physiologie de l'œil.

Toutefois, le terme de scotomisation, certes plus pertinent que celui de forclusion, a lui aussi ses limites. Après que Charcot l'a employé pour décrire des phénomènes hallucinatoires dans les années 1880, Freud a mis en doute l'opportunité de son importation dans le domaine psychanalytique, déclarant qu' "il éveille l'idée que la perception a été complètement balayée; comme dans le cas où une impression visuelle frappe la tache aveugle de la rétine ${ }^{39}$ ». Et, de fait, si l'on s'en tient à la définition médicale du terme, la tache aveugle envisagée comme "lésion rétinienne» est somme toute peu productive. De plus, la « tache aveugle » de l'image différée rétinienne n'est que temporaire - ce n'est pas une annihilation complète du champ visuel, mais une opacité persistante, une absence toujours présente. Roman construit sur une succession d'images rétiniennes, Triptyque redonne corps à l'impersonnel " on voit " à travers la présence de la scène absente, une scène qu'on ne peut pas voir mais qui laisse partout ses traces. Étymologiquement, scotomisation est issu du grec skotos (obscurité), mais c'est bien son contraire, la lumière, qui est à l'origine des taches aveugles. C'est pourquoi, même si la formule n'est pas des plus légère, on peut considérer que mimesis et anti-mimesis s'affrontent dans Triptyque sur les plans optique, matériel et figuratif à travers une logique de la "photo-scotomisation".

38. Le "trou " ou le vide causé par une image différée sur l'écran rétinien est temporaire, mais " un éclairement très intense peut même, s'il dure longtemps, amener une modification persistante de la partie correspondante de la rétine: [...] Ritter l'apprit à ses dépens après avoir regardé directement le soleil pendant 10 à 20 minutes " (Helmholtz, op. cit., \$23, p. 362)

39. Freud, «Fétichisme» (1927), cité par M. Jay, op. cit., p. 354. 